\title{
Anabases
}

ANABASES Traditions et réceptions de l'Antiquité

2 | 2005

Varia

\section{Tra religione e archeologia : Mystic Italy di}

M. Rostovtzeff

\section{Pier Giuseppe Michelotto}

\section{(2) OpenEdition}

Journals

Edizione digitale

URL: http://journals.openedition.org/anabases/1563

DOI: 10.4000/anabases.1563

ISSN: 2256-9421

\section{Editore}

E.R.A.S.M.E.

\section{Edizione cartacea}

Data di pubblicazione: 1 ottobre 2005

Paginazione: 53-77

ISSN: 1774-4296

\section{Notizia bibliografica digitale}

Pier Giuseppe Michelotto, «Tra religione e archeologia : Mystic Italy di M. Rostovtzeff », Anabases

[Online], 2 | 2005, Messo online il 01 juillet 2011, consultato il 20 octobre 2019. URL : http:// journals.openedition.org/anabases/1563; DOI : 10.4000/anabases.1563 


\title{
Tra religione e archeologia : Mystic Italy di M. Rostovtzeff
}

Pier Giuseppe Michelotto

\begin{abstract}
Con IL TITOlo di Mystic Italy veniva pubblicata, nei primi mesi del 1928, la raccolta delle tre Colver Lectures (I. "Mystic Italy", II. "Mystic Pompeii", III. "Mystic Rome") tenute da Rostovtzeff alla Brown University nella primavera del $1927^{1}$. Scopo del lavoro era delineare l'itinerario della "conversione" della cultura antica alla fede religiosa. Come l'Autore avvertiva nel "Preface", i contenuti del I e del III saggio riproducevano testi di conferenze del 1924, in parte (saggio III : "Mystic Rome") già pubblicati. Il saggio II ("Mystic Pompeii") invece, come dimostra l'apparato bibliografico contenuto nelle note (p. 159-170), fu concepito e comunque redatto nel 1927, forse con estremi ritocchi all'inizio del $1928^{2}$.

Mystic Italy rivela già prima facie la sua natura di opera composita, evidentemente costruita per successive aggregazioni di riflessioni e di convinzioni maturate (e in qualche caso mutate) tra la stagione di Madison e l'approdo a Yale, ma sedimentate a
\end{abstract}

1 M. Rostovtzeff, Mystic Italy, New York, 1927, p. XI. Il Copyright è del 1927 ; il "Preface" è tuttavia datato "January, 1928 ".

2 La citazione di " the excellent new chapter in F. Cumont, Les religions orientales, new French edition, 1928 " (RostOvTZEFF, Mystic Italy, p. 163 n. 3) non è necessariamente probante : dati i legami di amicizia tra i due studiosi, non è impossibile che Rostovtzeff abbia avuto modo di aver notizia del libro prima della stampa. Per la corrispondenza Cumont-Rostovtzeff cf. C. BonNET, La correspondance scientifique de Franz Cumont conservée à l'Academia Belgica de Rome, (Institut Historique Belge de Rome. Études de Philologie, d'Archéologie et d'Histoire Anciennes, XXXV), Bruxelles-Rome, 1997, p. 426-431 ; G. Bongard-Levin - Yu. Litvinenko - C. Bonnet - A. Marcone, "Aperçu préliminaire de la correspondance entre Franz Cumont et Mikhail Rostovtzeff", Bulletin de l'Institut Historique Belge de Rome 70 (2000), p. 349-412. 
partire almeno dal $1918^{3}$. Alla disomogeneità di Mystic Italy concorreva la stessa molteplicità dei punti di vista (economico-sociale, politico, psicologico, religioso) da cui Rostovtzeff procedeva nell'esame della conversione del mondo classico al misticismo realizzatasi alla svolta dell'èra volgare : una molteplicità che nelle intenzioni di Rostovtzeff era necessariamente destinata a convergere in una ricostruzione unitaria e nel coerente amalgama di uno scenario onnicomprensivo.

Rostovtzeff, consapevole delle insidie che costellavano il cammino in cui si avventurava, non solo si affrettava a premettere « my book must be regarded as an essay, not as a learned monograph ${ }^{4}$ ", ma - con prudente minimalismo - delimitava l'oggetto della trattazione e il proposito del volumetto a una " analysis of certain monuments of Pompeii and of Rome which reflect mystic tendencies in the population of these two places during the early Roman Empire 5 ".

Una sorta di disagio improntava l'atteggiamento di Rostovtzeff nei confronti dei temi religiosi e, più in generale, della storia delle religioni. Da un lato egli sempre si asteneva dal calarsi nei dibattiti teorici sottesi all'esame e all'uso di materiali religiosi ${ }^{6}$;

3 Penso soprattutto a M. RostovtzefF, La nascita dell'impero romano, Mosca, 1918 (in russo). Del saggio è in corso di stampa la traduzione italiana (di Aldo Ferrari) preceduta da una mia "Introduzione" e da un saggio di Emilio Gabba. Cf. J. ANDrEAU, "Antique, moderne et temps présent : la carrière et l'œuvre de Michel Ivanovič Rostovtseff (18701952)", "Introduction" a M.I. RostovTSEFF, Histoire économique et sociale de l'empire romain, traduction française, Paris, 1988, p. I-LXXXIV, in particolare p. XXI-XXII ; G. Bongard-Levin, "M.I. Rostovtzeff in England : a Personal Experience of West and East", in G.R. Tsetskhladze (a cura di), Ancient Greeks. West and East, Leiden, 1999, p. 1-45; G. Bongard-Levin, "Un scythe russe sur les rives de la Seine", Comptes rendus de l'Académie des inscriptions et belles lettres (1997), p. 863-879 ; G.W. BOWERSOCK, "The Social and Economic History of the Roman Empire by Michael Ivanovitch Rostovtzeff", Daedalus 103 (1974), p. 15-23 ; ID., "Rostovtzeff in Madison", The American Scholar 55 (1986), p. 391-400 ; M.A. WeS, Michael Rostovtzeff, Historian in Exile. Russian Roots in an American Context (Historia, Einzelschriften, 65), Stuttgart, 1990, p. 59-74. Rostovtzeff, Mystic Italy, p. XII. Cf. A. Momigliano, "In memoria di Michele Rostovtzeff (1870-1952)", Rivista Storica Italiana 45 (1953), p. 481-495, in particolare p. 490 = A. Momigliano, "Introduzione" a M. RostovtZefF, Storia economica e sociale del mondo ellenistico, traduzione italiana, I, Firenze, 1966 (rist. 1981), p. IX-XXIII, in particolare p. XVIII, definì Mystic Italy « semi-popolare ».

6 Cf. ad es. M. RostovtzefF, Dura-Europos and its Art, Oxford, 1938, p. 57. 
dall'altro egli teorizzava costantemente 7 l'incidenza primaria e strutturale, nel dipanarsi dei processi storici, dei fenomeni religiosi in quanto essi, nel loro manifestarsi, inerivano a quella mutazione di mentalità e a quell'evoluzione della psicologia collettiva in cui Rostovtzeff abitualmente inquadrava le sue rappresentazioni dell'ascesa e del declino della civiltà greco-romana. I due volti di Rostovtzeff si giustapponevano anche nel "Preface" di $S E H H W^{8}$ : mentre affermava la sua esigenza di storia totale e la sua incapacità di sopravvalutare, "in the Marxian fashion ", gli aspetti economico-sociali della vita umana, egli riconosceva di aver limitato la sua ricerca entro l'orizzonte economicosociale in quanto "I imagine that I am more competent in this field than in others ». In tal modo Rostovtzeff stesso apriva il varco alla critica rivoltagli da Momigliano, che tra l'altro scriveva : "Le idee politiche, religiose, morali dei Greci e dei Romani non lo hanno mai trattenuto a lungo. La crisi religiosa del Cristianesimo non lo ha mai fatto riflettere, almeno in pubblico, come storico ${ }^{9}$. " La considerazione di Momigliano sebbene espressa in forma tranchante - non era lontana dalla verità, anche perché riprendeva incertezze e dubbi più volte ed esplicitamente enunciati dallo stesso Rostovtzeff ${ }^{10}$. In una lettera a Cumont del 3 febbraio 1938, pubblicata nello splendido volume di C. Bonnet ${ }^{11}$, Rostovtzeff alludeva con fastidio al tema della Ingersoll Lecture del 1938, definendolo " cette malheureuse experience sur l'immortalité "; e a Cumont ribadì il proprio dilettantismo in materia storico-religiosa quando gli sottopose, nell'e-

7 Cf. M.I. RostovtzefF, "Il tramonto della civiltà antica”, Russkaja Mysl', 6-7 (1922), p. 190-214 ; 8-12 (1922), p. 3-36 = traduzione italiana in M.I. RostovTZEFF, Per la storia economica e sociale del mondo ellenistico romano. Saggi scelti, a cura di T. GNOLI J. Thornton, con "Introduzione" di M. MAZZA, Catania, 1995, p. 89-155 ; P.G. MichelotTo, "Italia 1923 : Sei lettere di Rostovtzeff a "Zveno" », in B. VirGILIO (a cura di), Studi ellenistici XVI, Pisa, 2005, p. 423-510, pubblicato (con varianti) anche in russo in G. BONGARD-LEVIN - Y. LiTVINENKO, La freccia partica, Mosca, 2003 (in russo), p. 636-680 ; nel corso del presente articolo verranno sempre citate le pagine della versione italiana. Cf. P.G. MichelotTo, "Momigliano, Rostovtzeff e la 'decadenza' dell'Impero romano : osservazioni", in Giornata Lincea in ricordo di Arnaldo Momigliano - Roma 22 aprile 1992 (Atti dei Convegni Lincei, 105), Roma, p. 21-48 ; ID., "Un'opera 'anomala' di M.I. Rostovtzeff", Acme 54, 2 (2001), p. 37-73.

8 M. RostovtzefF, The Social and Economic History of the Hellenistic World, Oxford, 1941 (rist. 1953), p. VII-VIII.

9 Momigliano, "In memoria", p. 494-495 = Momigliano, "Introduzione", p. XXIIXXIII.

10 Cf. e.g. M. RostovtzefF, "The mentality of the hellenistic world and the after-life", Harvard Divinity School Bulletin, Ingersoll Lecture (1935), p. 5-25.

11 Bonnet, Correspondance, p. 430. 
state 1927, la lettura del manoscritto di Mystic Italy ${ }^{12}$, ricevendone osservazioni e un moderato apprezzamento ${ }^{13}$.

Nelle note di "Mystic Pompeii" Rostovtzeff si limitava a ricordare lapidariamente che " the Hellenistic mystery religions are treated mostly in connection with early Christianity 14 ", astenendosi da qualsiasi approfondimento. In definitiva, in Mystic Italy (e ancora in "The Mentality" del 1938), Rostovtzeff si sottraeva alla discussione sulle origini cristiane, sul problema dell'eventuale rapporto tra mistero pagano e mistero cristiano (e tra salvezza pagana e salvezza cristiana) ${ }^{15}$, sulla accostabilità (se non sull'interazione) tra sacramentalismo pagano e sacramentalismo cristiano, sulla relazione (e sui possibili livelli, tempi e modi di questa relazione) tra ellenismo e giudaismo che inevitabilmente doveva culminare in una valutazione della formazione della teoresi paolina, come mostrava, tra gli altri (e penso soprattutto a Wendland, a Reitzenstein, a Loisy), il pressoché contemporaneo "Early Gentile Christianity" di Nock ${ }^{16}$.

In particolare il Rostovtzeff di Mystic Italy - e in generale della produzione antecedente all'esperienza di Dura ${ }^{17}$ - sorvolava sui problemi dell'eventuale apporto giudaico alle origini cristiane e degli influssi ellenistici eventualmente rintracciabili nel giudaismo precristiano, comunque astenendosi da radicali prese di posizione alla maniera di Zielinski ${ }^{18}$. Del resto, ancora nel $1941^{19}$, nel momento in cui constatava

12 Da Rostovtzeff, Mystic Italy, p. XV, emerge che Rostovtzeff sottopose alla lettura di Cumont solo i primi due saggi ("Mystic Italy", "Mystic Pompeii").

13 Devo l'informazione alla gentilezza di Corinne Bonnet.

14 RostovtzefF, Mystic Italy, p. 163 n. 3.

15 Mi limito a rammentare l'intervento di R. PETTAZZONI, "Les Mystères grecs et les religions à mystères de l'Antiquité. Recherches récentes et problèmes nouveaux", Cahiers d'Histoire Mondiale II 2 (1954), p. 303-312 ; 661-667 = traduzione italiana in "Appendice" a R. PetTAZzoni, I misteri. Saggio di una teoria storico-religiosa, Cosenza, $1997^{2}$ (I edizione 1924), p. 223-242 e la ben nota polemica che, a proposito delle religioni misteriche di età ellenistica, oppose R. REITZENSTEIN, Die Hellenistischen Mysterienreligionen nach ibren Grundgedanken und Wirkungen, Leipzig-Berlin, 19273, al MEYER di Ursprung und Anfänge des Christentums, III, Stuttgart-Berlin, 1923.

16 A.D. NoCK, "Early gentile christianity and its hellenistic background", in A.E.J. RAWLINSON (a cura di), Essays on the Trinity and the Incarnation, London, 1928, p. 51-156 = A.D. NocK, Essays on Religion and the Ancient World, I, Oxford, 1972, p. 49-133.

17 Per il dopo-Dura cf. ad es. RostovtZeFf, Dura-Europos, p. 100-134 ; ID., "How archaeology aids history", The Yale Review 31 (1941), p. 713-729.

18 T. ZiELINSKI, La Sibylle. Trois essais sur la religion antique et le christianisme, Paris, 1924 ; cf. Andreau, "Antique, Moderne", p. VI. Rostovtzeff, Mystic Italy, p. 63 segnalava anche " the brilliant Russian book of T. Zielinski, The Religion of the Hellenistic Period, S. Petersburg, 1922 », mentre stranamente non citava T. ZIELINSKI, The Religion of Ancient Greece. An Outline, Oxford-London, 1926 (che a p. 152-156 trattava dei "misteri orfici"), versione inglese - tratta dall'originale polacco - cui Rostovtzeff aveva in qualche misura collaborato, come risulta dalla "Note" di p. 5. RostovtZEFF, "How Archaeology", p. 727-729. 
la compresenza, in Dura, di una sinagoga e di un edificio di culto cristiano, il vecchio Rostovtzeff riconosceva come insolubile, pur se ineludibile, il problema del rapporto tra il giudaismo e le origini cristiane.

In "Mystic Rome 20" Rostovtzeff accennava piuttosto oscuramente a " mysteries... of the Jewish Jahve " e a "Greco-Semitic "mysteries" of the Christians "; in "Mystic Italy" (I saggio) e in "Mystic Rome 21" riconosceva, nelle affinità di linguaggio accomunanti tutte le religioni mistiche, l'avvenuta costituzione di una koiné che veniva a comprendere, in "mutual interpenetration ", anche il Cristianesimo ${ }^{22}$, in particolare quelle facies di Cristianesimo non canonico che egli ravvisava nelle raffigurazioni pittoriche delle tombe scoperte sotto la Chiesa di S. Sebastiano e in quelle - davvero enigmatiche - dell'Ipogeo degli Aurelii, adepti di una delle sette cristiane eretiche che egli definiva genericamente " gnostiche 23 ». A dottrine cristiane eretiche e a teorie gnostiche si solevano del resto attribuire le pitture dell'Ipogeo della Via Latina ${ }^{24}$, accomunato da Rostovtzeff alla Tomba di Vincentius e Vibia e al sepolcro dei Nasonii nel suo excursus sui monumenti attestanti la koiné mistica romana ${ }^{25}$.

Dall'evidenza della "mutual interpenetration ", tuttavia, mai Rostovtzeff fu indotto - se non fuggevolmente in "The Mentality 26" - alla riflessione sul problema e sul concetto stesso di sincretismo ; mai egli tentò di offrire una definizione di mistico o approfondì il rapporto tra il mistico e il mistero ${ }^{27}$; mai si impegnò a discutere sull'eventuale carattere sacramentale dei riti misterici, in ciò distanziandosi nettamente, ad esempio, da quel Vittorio Macchioro cui pure sovente faceva riferimento in Mystic Italy. In definitiva, il Rostovtzeff di Mystic Italy rifletteva, senza però esserne voce costitutiva,

20 RostovtzefF, Mystic Italy, p. 110.

21 RostovtzefF, Mystic Italy, p. 21 ; 144-155, dove è centrale la discussione sull'Ipogeo di Vibia.

22 RostovtzefF, Mystic Italy, p. 146 : «And among the mystery religions Christianity is not an exception ".

23 Rostovtzeff, Mystic Italy, p. 148-155. Cf. le oscillazioni di F. CoARelli, Guida archeologica di Roma, Milano, $1975^{2}$, p. 210-211 ; ID., Roma, Roma-Bari, 198532, p. 221-224 e ID., Roma, Milano, $1997^{3}$, p. 235. Cf. anche M. BoRDA, La pittura romana, Milano, 1958, p. 319, per l'ipotesi "montanista". Su questi temi - e sul relativo dibattito - mi sono a lungo soffermato in MichelotTO, Italia 1923, p. 430-434; 453-456; 472-476.

24 Così, ad es., da parte di R. PARIBENI, "Culti e religioni in Roma imperiale secondo recenti scoperte archeologiche”, Atene e Roma 1 (1920), p. 169-183, in particolare p. 172, che riprendeva un suggerimento di Marucchi. Per la Via Latina, Rostovtzeff citava solo E.L. Wadsworth, Stucco Reliefs of the First and Second Centuries Still Extant in Rome (American Academy in Rome, IV), Roma, 1924.

25 RostovtzefF, Mystic Italy, p. 145.

26 RostovtzefF, “The Mentality”, p. 13.

27 Cf. W. BURKERT, Antichi culti misterici, traduzione italiana, Roma-Bari, 1991, p. 12-18. 
il vivacissimo dibattito storico-religioso del primo trentennio del Novecento, che proprio tra il '20 e il ' 30 giunse a un momento di svolta ${ }^{28}$.

Naturalmente il fatto di muoversi ai margini del dibattito propriamente storicoreligioso non impediva a Rostovtzeff di manifestare inclinazioni e avversioni : ad esempio egli non dimostrava interesse specifico per la metodologia filologizzante dei vari Reitzenstein, Loisy e dello stesso giovane Nock, che proprio negli anni della concezione dei saggi di Mystic Italy più volte scrisse sulla Basilica sotterranea presso Porta Maggiore sulla quale si diffondeva anche il Rostovtzeff di "Mystic Rome 29". E Rostovtzeff neppure dimostrava di dipendere da autori complessi, esponenti di una stagione panorfica $^{30}$, quali furono R. Eisler, V. Macchioro, O. Kern e A. Boulanger. Una palmare riprova della sua sostanziale estraneità al dibattito storico-religioso è data da una citazione-satura di Wissowa, Toutain e di Cumont ${ }^{31}$; citazione che avrebbe doverosamente richiesto dei distinguo ${ }^{32}$. In definitiva, a lasciare tracce visibili in Mystic Italy fu solo l'ormai trentennale dear friend Franz Cumont (e in particolare il Cumont di After Life in Roman Paganism 33), che nel "Preface 34" Rostovtzeff definiva " the greatest living authority on the history of ancient religions ». E forse Cumont sarebbe stato dedicatario del libro se non fosse intervenuta l'improvvisa morte del comune amico Walther Amelung 35.

28 Alle porte c'erano Der Glaube der Hellenen di Wilamowitz (1931-1932), Conversion di Nock (1933) ; poco più avanti Festugière e l' "orfismo rivisitato" di Guthrie e di Nilsson (1935). Per un orientamento di carattere generale e per la bibliografia, mi limito a ricordare L. Salvatorelli, "Da Locke a Reitzenstein. L'indagine storica delle origini cristiane", Rivista Storica Italiana 45 (1928), p. 341-369 ; 46 (1929), p. 5-66 ; cf. M. MAZZA, "Introduzione" ad A.D. NOCK, La conversione. Società e religione nel mondo antico, traduzione italiana, Roma-Bari, 1985, p. VII-XXXVII ; M. MAZZA, "Le religioni dell'impero romano. Premesse ad una considerazione storica della religiosità ellenistico-romana", in Storia Letteratura e Arte a Roma nel secondo secolo dopo Cristo (Atti del Convegno di Mantova, 8-9-10 ottobre 1992), Firenze, 1995, p. 109-138 ; A. Momigliano, Saggi di storia della religione romana. Studi e lezioni 1983-1986, a cura di R. Di DonATO, Brescia, 1988, p. 67-86 ; B.M. METZGER, "Considerations of methodology in the study of the mistery religions and early Christianity”, Harvard Theological Review 48 (1955), p. 1-20.

32 R. MacMullen, Paganism in the Roman Empire, New Haven-London, p. 116-117 ; cf. Momigliano, Saggi di storia, p. 102-106 ; 148-149.

33 F. Cumont, After Life in Roman Paganism, New York, 1922 (rist. 1959).

34 RostovtzefF, Mystic Italy, p. XV.

35 Alla morte di Amelung accenna una lettera di Rostovtzeff a Cumont del 18 settembre 1927 : devo la notizia alla cortesia di Corinne Bonnet. 
Da quanto esposto si comprende bene perché, a interrompere di tanto in tanto il pressoché generale oblio in cui ben presto cadde Mystic Italy, intervennero con maggiore frequenza archeologi e storici dell'arte antica come K. Schefold 36 che non storici delle religioni come Nilsson, o Nock o lo stesso Macchioro 37.

Pur essendo naturalmente portato alla " lecture réaliste et sociale " del monumento antico ${ }^{38}$, Rostovtzeff non escludeva affatto un' esegesi del monumento in chiave simbolica e, mentre invitava a non ricadere nel " fatal error of Creuzer ", affermava che " in many cases there is no other way to explain the monuments except by invoking religion and symbolism 39 ». In questo aspetto, per cui Rostovtzeff poteva essere accostato a Cumont e a Rodenwaldt, K. Schefold colse esattamente l'importanza e la novità del volumetto ${ }^{40}$. Di più : se l'interpretazione della megalografia della Villa dei Misteri era improntata, come si vedrà, a un simbolismo sostanzialmente temperato, l'entusiasmo ricostruttivo dei pinakes della Casa del Criptoportico (detta anche Casa "Omerica") spingeva l'esegesi simbolica di Rostovtzeff a un limite quasi "macchioriano" di complessità.

Il secondo saggio di Mystic Italy coronava la più che trentennale stagione pompeiana di Rostovtzeff, precocemente fiorita nel corso degli studi universitari $41 \mathrm{e}$ arricchitasi grazie alla frequentazione dell' Istituto Archeologico Germanico di Roma 42 e

36 K. SCHEFOLD, La peinture pompéienne. Essai sur l'évolution de sa signification, traduzione francese di J.-M. Croisille (Collection Latomus, 108), Bruxelles, 1972, p. 33-34.

37 M.P. Nilsson, The Dionysiac Mysteries of the Hellenistic and Roman Age (Skrifter Utgiuna av Svenska Institutet I Athen, V), Lund, 1957, p. 67 n. 1 ; 76-88 ; 112 n. 14 ; 126 ; Nock, Conversione, p. 234 ; V. MACCHIORO, Zagreus. Studi intorno all'orfismo, Firenze, $1930^{2}$, p. $63 ; 161$ n. 6.

38 BONGARD-LEVIN, "Un scythe russe”, p. 878. Bongard-Levin si riferiva in particolare all'interpretazione delle "images funéraires".

39 Rostovtzeff, Mystic Italy, p. 23. Per Creuzer cf. ultimamente F. Morelli, Lo sguardo da Oriente. Simbolo, mito e grecità in Friedrich Creuzer, Milano, 2000.

40 SCHEFOLD, La peinture, p. 34.

41 C.B. Welles, "Bibliography - M. Rostovtzeff", Historia 5 (1956), p. 358-381, in particolare p. $360 \mathrm{n}^{\circ} 1-2$; J.F. GILliAM, "Addenda to the Bibliography of M.I. Rostovtzeff”, Historia 36 (1987), p. 1-8, in particolare p. $1 \mathrm{n}^{\circ} 2$; cf. le p. 648-649 della "Bibliographie" rostovtzeviana (p. 648-675) curata da J. ANDREAU nell'edizione francese di SEHRE, ricordata supra alla n. 3 ; cf. la p. 201 della bibliografia rostovtzeviana (p. 200-230) pubblicata da V. Zuev in G. Bongard-LeVin - Yu. LitvinenKo (a cura di), Romanzo scita, Mosca, 1997 (in russo). Si veda anche A. MArCone, "Introduzione” ad A. MARCone (a cura di), Rostovtzeff e l'Italia (Incontri perugini di Storia della storiografia antica e sul mondo antico, IX, Atti del Convegno di Gubbio, 25-27 maggio 1995), Napoli, 1999, p. 9-22 ; ANDREAU, "Antique, moderne", p. XLV.

42 A. Marcone, "Michele Rostovtzeff e l'Istituto Archeologico Germanico di Roma : la corrispondenza con Christian Hülsen (1894-1927)", Critica Storica 25 (1988), p. 339350 ; ID., "Pietroburgo-Roma-Berlino : l'incontro di M.I. Rostovtzeff con l'Altertumswissenschaft tedesca", Historia 41 (1992), p. 1-13. 
in particolare di Christian Hülsen e Walther Amelung 43. Dalla frequentazione dell'Istituto Germanico nacquero lo studio del 190444 sui paesaggi pompeiani e le ville romane e il più ampio saggio, pubblicato in russo nel 1908 e in tedesco nel 1911, sul paesaggio architettonico ellenistico-romano 45 ; sarebbe seguita la sintesi - di carattere più generale - di “Ancient Decorative Wall-Painting 46". Né va dimenticata, specie in rapporto a "Mystic Rome", la frequentazione di epigrafisti e antiquari italiani che, in tempi diversi, ebbero nel Dizionario epigrafico del De Ruggiero uno dei punti di aggregazione ${ }^{47}$, da Vaglieri ${ }^{48}$ a Breccia a Paribeni, dal 1919 Soprintendente agli Scavi di Roma ${ }^{49}$. Terreno ancora inesplorato rimane quello degli eventuali rapporti di Rostovtzeff con gli archeologi romani, dal Marucchi al Mancini, che con il Fornari provvidero agli scavi di S. Sebastiano ${ }^{50}$, con il Gatti e soprattutto con il Bendinelli, che si occupò degli Ipogei della Via Trionfale ${ }^{51}$, dell'Ipogeo degli Aurelii 52 e - quel che qui

43 RostovtzeFF, Mystic Italy, p. XIV-XV ; cf. Bonnet, Correspondance, p. 428.

44 M. RostovtzefF, "Pompeianische Landschaften und römische Villen", Jahrbuch des Deutschen Archäologischen Instituts 19 (1904), p. 103-126.

45 M. RostovTzeFF, "Die hellenistisch-römische Architekturlandschaft", Mitteilungen des Deutschen Archäologischen Instituts. Römische Abteilung 26 (1911), p. 1-185. R. LING, "Studius and the Beginnings of Roman Landscape Painting", Journal of Roman Studies 67 (1977), p. 1-16, lo giudicava (p. 2) " for its time, a remarkable and fundamental study ». M. Rostovtzeff, "Ancient Decorative Wall-Painting”, Journal of Hellenic Studies 39 (1919), p. 144-163.

47 G. SALMERI, "Epigrafia e storia antica nel Mediterraneo : il 'caso' italiano", in V. LA Rosa (a cura di), L'archeologia italiana nel Mediterraneo fino alla seconda guerra mondiale, (Centro di Studi per l'Archeologia greca C.N.R.-Catania, Atti del Convegno di Studi di Catania, 4-5 novembre 1985), Catania, 1986, p. 203-229 ; A. MARCONE, "Rostovtzeff in Italia”, Annali della Scuola Normale Superiore di Pisa IIIa ser., 24, 4 (1994), p. 923-938; ID., "Introduzione", p. 16-19; M. CAGNETTA, "Rostovtzeff in Italia : mediazioni culturali e vicende editoriali", in MARCONE (a cura di), Rostovtzeff e l'Italia, p. 161-185.

48 MichelotTo, "Italia 1923", p. 484-485 n. 93.

49 D. MANACORda - R. TAMASSiA, Il piccone del regime, Roma, 1985, p. 23 ; già in D. MANACORDA, "Per un'indagine sull'archeologia italiana durante il ventennio fascista", Archeologia Medievale 9 (1982), p. 443-470, in particolare p. 453-454 ; M. BARBANERA, L'archeologia degli italiani. Storia, metodi e orientamenti dell'archeologia classica in Italia, Roma, 1998, p. 225-226.

50 G. MAncinI, "Scavi sotto la basilica di S. Sebastiano sull'Appia Antica”, Notizie degli Scavi di Antichità Va ser., 20 (1923), p. 3-79 ; O. MARUCCHI, "Nota sulle memorie cristiane esplorate nello scavo di S. Sebastiano dalla Commissione di Archeologia Sacra”, Notizie degli Scavi di Antichità Va ser., 20 (1923), p. 80-103.

51 G. BendinelLI, "Ipogei sepolcrali scoperti presso il Km. IX della Via Trionfale (Casale del Marmo)", Notizie degli Scavi di Antichità Va ser., 19 (1922), p. 428-449.

52 G. Bendinelli, "Il monumento sepolcrale degli Aureli al viale Manzoni in Roma", Monumenti Antichi - Accademia dei Lincei 28 (1923), p. 289-520 ; cf. RostovtZeFf, Mystic Italy, p. 175 n. 15. 
più conta - della cd. Basilica di P.ta Maggiore 53. Per ciò che concerne i rapporti con gli archeologi italiani che lavorarono a Pompei, Rostovtzeff ebbe contatti con Vittorio Spinazzola 54, ma soprattutto con Della Corte e, almeno dal 1924, con Maiuri. Nel 1931 - tre anni dopo l'uscita di Mystic Italy - Maiuri pubblicò un'opera di grande chiarezza descrittiva sulla Villa dei Misteri 55 nella quale si misurava con le tesi di Macchioro (il principale bersaglio polemico), Comparetti, Rizzo e soprattutto con l'esegesi, decisamente sopravvalutata, della Bieber, ma non con la ricostruzione tentata da Rostovtzeff in "Mystic Pompeii 56". Nel silenzio di Maiuri (e di Macchioro) su Mystic Italy - destinato ad essere sporadicamente interrotto dalle critiche, a volte severe, che lo stesso Maiuri 57

53 G. Bendinelli, "Il Mausoleo sotterraneo altrimenti detto Basilica di Porta Maggiore", Bullettino della Commissione Archeologica Comunale in Roma 50 (1922), p. 85-126; prima di Bendinelli scrissero sulla cd. Basilica anche Gatti e Fornari : E. GATTI - F. FORNARI, "Brevi notizie relative alla scoperta di un monumento sotterraneo presso Porta Maggiore", Notizie degli Scavi di Antichità Va ser., 15 (1918), p. 30-39 (Gatti) ; $39-53$ (Fornari). Rostovtzeff non poteva conoscere, al momento della concezione di "Mystic Rome", il saggio "definitivo" del Bendinelli sul monumento (cf. G. BENDINELLI, "Il monumento sotterraneo di Porta Maggiore. Contributo alla storia dell'arte decorativa augustea", Monumenti Antichi - Accademia dei Lincei 31 [1926], p. 601-848) e comunque non dava segno di conoscerlo neppure al momento di licenziare l'intero volume di Mystic Italy.

54 RostovtzefF, "Die hellenistisch-römische", p. 4. Cf. MichelotTo, "Italia 1923", p. 444 e le note a p. 495-504.

55 A. Maiuri, La Villa dei Misteri, Roma, 1931. Ho letto la seconda edizione del 1947.

56 V. Macchioro, Zagreus. Studi sull'orfismo, Bari, 1920 (una seconda edizione, profondamente rinnovata, dello Zagreus, uscì a Firenze nel 1930 : cf. supra n. 37) ; ID., The Villa of the Mysteries in Pompei, Napoli, s.d. ; D. ComparetTi, Le nozze di Bacco ed Arianna. Rappresentazione pittorica spettacolosa nel triclinio di una villa suburbana di Pompei, Firenze, 1921 ; G.E. Rizzo, "Dionysos mystes. Contributi esegetici alle rappresentazioni di misteri orfici", Memorie della Reale Accademia di Archeologia, Lettere e Belle Arti Società Reale di Napoli 3 (1918), p. 39-102 (= p. 3-66 dell'estratto) : il saggio di Rizzo fu licenziato nella primavera del 1914 ; M. BIEBER, "Die Mysteriensaal der Villa dei Misteri", Jahrbuch des Deutschen Archäologischen Instituts 43 (1928), p. 298-330. Neppure Bendinelli menzionò l'opera di Rostovtzeff quando offrì la sua eccentrica interpretazione della megalografia della Villa dei Misteri : G. BENDineLLI, "Il fregio dionisiaco della Villa dei Misteri a Pompei", Miscellanea della Facoltà di Lettere e Filosofia di Torino 1 (1936), p. 17-56.

57 A. MaIuri, "Studi e ricerche intorno alla 'Casa del Criptoportico' sulla Via dell'Abbondanza”, Notizie degli Scavi di Antichità VIa ser., 9 (1933), p. 252-276 : le critiche a Rostovtzeff a p. 263 nn. 1 e 2 ; 264 n. 1 ; 265 n. 2 ; 266. Già all'inizio dell'articolo (p. 253 n. 2), Maiuri liquidava duramente l'interpretazione rostovtzeviana della Casa del Criptoportico, "che vuole essere un geniale ma non convincente " tentativo di esegesi mistica e dionisiaca. Rostovtzeff, viceversa, ebbe sempre parole di gratitudine e di apprezzamento per Maiuri, che egli ringraziava per vari motivi nel "Preface" di Mystic Italy: RostovtzefF, Mystic Italy, p. XIII. Cf. Michelotto, “Un'opera 'anomala”, p. 50-51. 
e lo Spinazzola 58 rivolsero alle interpretazioni rostovtzeviane della Casa del Criptoportico - si rifletteva una più generale tendenza, rappresentata dall'indifferenza pressoché assoluta dell'archeologia italiana tra le due guerre nei confronti della straordinaria personalità scientifica di Rostovtzeff 59 .

L'attenzione della critica a Mystic Italy non fu comunque tiepida solo in Italia : le recensioni al volumetto - per lo più brevi segnalazioni - si contano sulle dita di una mano e non rendono adeguatamente conto dei meriti - oltre che degli effettivi limiti strutturali 60 - di questo scritto anomalo. In particolare non fu sottolineata l'originalità (e l'aleatorietà) del saggio "Mystic Pompeii", attestante lo sforzo di racchiudere in un unico orizzonte interpretativo documenti pittorici tanto diversi come la megalografia della Villa dei Misteri e i pinakes della Casa "Omerica".

Indubbiamente meno fantasiosa risultava la lettura rostovtzeviana della cd. Basilica di Porta Maggiore su cui si imperniava lo scenario di "Mystic Rome". Rostovtzeff, ormai convinto della esattezza dell'esegesi di Cumont ${ }^{61}$ e di Carcopino ${ }^{62}$, ricordava la sua originaria interpretazione dell'edificio - peraltro non apprezzata da alcuno studioso - come " a hall for recreation or recitations 63 » : un'ipotesi per la verità condivisa, se non addirittura suggerita, dal suo amico Christian Hülsen ${ }^{64}$, il quale

58 V. Spinazzola, Pompei alla luce degli scavi nuovi di Via dell'Abbondanza (anni 19101923), Roma, 1953 (opera postuma a cura di S. AurigemmA), nel vol. I, a partire da p. 435, più volte alludeva pesantemente nel testo (ad es. p. 504-519) alla erroneità delle interpretazioni rostovtzeviane intorno alla Casa del Criptoportico. Le critiche si esplicitarono nelle "Note" (p. 665-669, nn. 367-397) curate da A. LeVI, S. Aurigemma e D. FACCENNA.

59 Negli "Interventi" di chiusura in LA Rosa (a cura di), L'archeologia italiana, p. 250, M. TORELli notava con esattezza : "Oggi... ricordavo come fosse passata del tutto inosservata presso gli archeologi italiani la presenza a Roma di Michael Rostovtzeff e come tutto questo non abbia avuto veramente nessun segno prima del periodo dopo la Seconda Guerra Mondiale ».

60 Nemmeno R.C. Flickinger, recensione a Rostovtzeff, Mystic Italy, in The Classical Journal 26 (1930), p. 153-155, che pure si avvide di qualche importante contraddizione.

61 F. Cumont, "La basilique souterraine de la Porta Maggiore", Revue Archéologique 8 (1918), p. 52-75 (= p. 1-23 dell'estratto). Cf. E. STRONG - N. Jolliffe, "The stuccoes of the underground basilica near the Porta Maggiore", Journal of Hellenic Studies 44 (1924), p. 65-111 ; J. BousQUET, "Les confrères de la Porte Majeure et l'arithmologie pythagoricienne", Revue des études grecques 64 (1951), p. 466-471; fondamentale, come è noto, fu l'identificazione di Saffo nella protagonista della scena dell'abside da parte di C.D. CuRTIS, "Sappho and the Leaucadian Leap", American Journal of Archaeology 24 (1920), p. 146-150.

62 RostovtzefF, Mystic Italy, p. 132-143 ; 173 n. 4 ; C.H. MoOre, recensione a RostovtzefF, Mystic Italy, in The Classical Weekly 22 (1929), p. 104.

63 RostovtzefF, Mystic Italy, p. 131 ; cf. MichelotTo, "Italia 1923", p. 429-430 ; 469472 , nn. 26-38 e soprattutto la "lettera" rostovtzeviana ivi pubblicata alle p. 450-453.

64 Chr. HÜlsEN, “Die Ausgraben in Rom”, XX Jahrhundert III 3 (29 gennaio 1921), p. 4852. 
tuttavia (come ricordò Carcopino ${ }^{65}$ ) era in precedenza andato molto vicino all'idea della destinazione funeraria del monumento poi fermamente sostenuta dal Bendinelli 66. Seguendo un percorso opposto a quello di Hülsen, Rostovtzeff si accostò comunque, non senza incertezze, alla tesi funeraria, che in maniera diversa influenzò numerosi studiosi 67 e fu riportata in auge - non senza trovare forti opposizioni 68 - dal Bastet ${ }^{69}$ prima di essere accolta da K. Schefold e, sia pur dubitativamente, da Filippo Coarelli ${ }^{70}$. Abbandonata la spiegazione « gioiosa » $\mathrm{e}$ " laica » della Basilica, Rostovtzeff sembrava incapace non solo di formulare un'ipotesi originale sull'edificio, ma anche di seguire con coerenza le ipotesi altrui : verosimilmente influenzato da Cumont e forse da Hülsen (meno probabilmente da Bendinelli), nell' "Augustus " del '22 accennava alla Basilica di Porta Maggiore con la prudenza - e l'incongruenza - di un giudizio che voleva essere salomonico ( be it a sanctuary of a mystical sect or a grave of an adherent of such a sect $\left.{ }^{71}\right)$ ), ulteriormente sfumato e arricchito nella versione tedesca dell'articolo ( ob es nun das Heiligtum einer mystischen Sekte sei oder die Grabstätte oder Grabtempel eines Anhängers solch einer Sekte $72 »)$. Lidea mistico-funeraria del "Grabtempel » dovette affascinare Rostovtzeff negli anni 1923/24, dato che anche in

65 J. Carcopino, La basilique pythagoricienne de la Porte Majeure, Paris, 1927, p. 83, n. 1. Cito dalla X edizione del 1944.

66 Cf. supra n. 53.

67 Una delle prime "messe a punto" fu quella di STRONG - Jolliffe, “The Stuccoes", p. 6670 .

68 Cf. ad esempio C.C. VAN ESSEN, recensione a F.L. BASTET, De datum van het grote Hypogaeum bij de Porta Maggiore te Rome, Leiden, 1958, in Mnemosyne s. IV, 13 (1960), p. 277-280.

69 BASTET, De datum, p. 39. Per una più ampia discussione cf. Michelotto, "Un'opera 'anomala', p. 52-56.

70 SCHEFOld, La peinture pompéienne, p. 87 ; COARELl, Guida archeologica, p. 213-216; ID., Roma, $1985^{3}$, p. $228-231$; ID., Roma, $1997^{3}$, p. 236-239 ; con prudenza analoga a quella di Coarelli, anche B. ANDREAE, L'art romain, Paris, 1973 (19982), p. 136, ritiene "vraisemblable» la destinazione funeraria ; analogamente J. PrIEUR, La mort dans l'antiquité romaine, Ouest France, 1986, p. 61 ; 139. Per la datazione della Basilica, ricordo che - tramontate le datazioni "basse" di R. LANCIANI, "Il santuario sotterraneo recentemente scoperto ad Spem Veterem", Bullettino della Commissione Archeologica Comunale in Roma 46 (1918), p. 69-84, in particolare p. 83 (età adrianea) e di G. BAGNANI, "The subterranean Basilica at Porta Maggiore”, Journal of Roman Studies 9 (1919), p. 78-85, in particolare p. 84 (" end of the first century») - il BASTET, De datum, p. 110 (ca. 20 d.C.) e ANDREAE, L'art romain, p. 137 (però cf. p. 497) propendono per l'età tiberiana, mentre COARElli, Roma, $1997^{3}$, p. 239 si spinge a proporre l'età "tardoaugustea o tiberiana ».

71 M. Rostovtzeff, "Augustus", Classical Studies, s. II, Univ. of Wisconsin Studies in Language and Literature 15 (1922), p. 132-147, in particolare p. 137.

72 M. Rostovtzeff, "Augustus", Mitteilungen des Deutschen Archäologischen Instituts. Römische Abteilung 38/39 (1923/24), p. 281-299, in particolare p. 284 ; sulle oscillazioni interpretative di Rostovtzeff cf. MicHELOTTO, "Italia 1923”, p. 427-430. 
"Mystic Italy" (saggio I) audacemente accostava la Basilica di Porta Maggiore al "gravetemple» (fanum) voluto da Cicerone in memoria della diletta figlia Tullia ${ }^{73}$.

Solo a partire da "Mystic Rome", bandendo ogni indugio e sposando l'intuizione cumontiana, Rostovtzeff definì la Basilica « a shrine, a place of worship and prayer, a place where a small community of mystae held its meetings... a religious and not a secular one, a place of devotion and not of rest and amusement ", " not a sepulchre itself 74 "; e ancor più esplicitamente in "Mystic Pompeii" : "Certainly a shrine, the church of a mystic community, probably Pythagorean, as Cumont suggested and Carcopino now has proved ${ }^{75}$. " Né Rostovtzeff fu l'unico studioso "convertito" dall'intuizione di Cumont : il Nock, che nel '24 con giovanile insolenza aveva irriso all' "ingenuità " di Cumont e aveva sostenuto l'appartenenza della Basilica a una sodalità orfico-dionisiaca $^{76}$, nel 1928, al tempo dell'uscita di Mystic Italy, si accodava ai sostenitori del neopitagorismo dell'edificio ${ }^{77}$, constatando - come il Rostovtzeff di "Mystic Pompeii 78" - che " at this time " (cioè intorno alla metà del I sec. d.C.) "Orphic ideas flourished in Neopythagorean circles particularly 79 ». Anche Rostovtzeff, adeguandosi all'allora communis opinio, riteneva che il sottofondo orfico, intridendo in varia misura credenze e filosofie religiose, costituisse una sorta di termine medio in grado di accomunare "mondi culturali e processi storici diversi 80 ", un elemento trasversale - e interclassista - tendente ad avvicinare, nella costituenda koiné mistica, le diverse esperienze religiose, dalle più elitarie (come il Neopitagorismo ${ }^{81}$ ) alle più popolari : se in

73 Rostovtzeff, Mystic Italy, p. 17. Cf. D. FeENEY, Letteratura e religione nell'antica Roma. Culture, contesti e credenze, traduzione italiana, Roma, 1999, p. 38.

74 RostovtzeFF, Mystic Italy, p. 131-132.

75 Rostovtzeff, Mystic Italy, p. 40 ; Macchioro, Zagreus, $1930^{2}$, p. 173. Cf. S. Reinach, recensione a RostovtzefF, Mystic Italy, in Revue Archéologique 28 (1928), p. 168 e n. 1.

76 A.D. Nock, "The historical importance of cult-associations", Classical Review 38 (1924), p. 105-109, in particolare p. 106-109 ; cf. ID., "Notes on beliefs and myths", Journal of Hellenic Studies 46 (1926), p. 47-53.

77 NocK, "Early Gentile", p. 65 = ID., Essays, p. 60 e n. 36. Si noterà che il riavvicinamento a Cumont si accompagnava a una presa di distanze dalle conclusioni di E. Strong e N. Jolliffe (Nock, ibid.). Cf. A.D. Nock, "Greek magical papyri", Journal of Egyptian Archaeology 15 (1929), p. 219-235, in particolare p. 227 = ID., Essays, p. 176-194 (con tagli) ; ID., "La religione romana dalla fine della repubblica al principato di Nerone", The Cambridge Ancient History, vol. X, 2, traduzione italiana, Milano, 1968, p. 681-727 ; 1143-1148; 1181-1183; in particolare p. 715 e 1147 , n. 95.

78 RostovtzeFf, Mystic Italy, p. 33-34.

79 NocK, "Early Gentile", p. 65 = ID., Essays, p. 60, n. 36. Cf. W. BURKERT, Lore and Science in Ancient Pythagoreanism, english translation, Cambridge Mass., 1972, p. 125.

80 U. BIANCHI, La religione greca, Torino, 1975 (rist. 1992), p. 225.

81 "Gruppuscolo integralista, monarchico e "autoritarista" " è definito da P. VEYNE, I misteri del gineceo, traduzione italiana, Roma-Bari, 2000, p. 112. 
"Mystic Italy" (saggio I), utilizzando sempre schemi cumontiani, Rostovtzeff constatava la presenza di motivi orfici nelle credenze dei vari strati sociali e intellettuali dell'ultimo secolo della repubblica ${ }^{82}$, nel più articolato quadro offerto nell' excursus iniziale di "Mystic Pompeii" osservava l'incidenza dell'orfismo nella vita spirituale dei mondi greco, magnogreco, siceliota e italico, ma soprattutto ne constatava gli importantissimi esiti ellenistici ${ }^{83}$. Ovviamente si guardava bene sia dall'abbordare temi roventi come lo sviluppo dell'orfismo o l'esistenza o meno di un puro orfismo inteso come " distinct religion 84 ", sia dal considerare l'orfismo un antecedente diretto del cristianesimo o addirittura, alla maniera di Zielinski e di Macchioro ${ }^{85}$, un elemento costitutivo di concezioni cristiane e in particolare dell'escatologia cristiana.

Rostovtzeff non si mostrava corrivo ad accogliere soluzioni radicalmente orfiche al dilemma posto dalla megalografia della Villa dei Misteri ${ }^{86}$. A suo avviso, il fregio pittorico aveva la funzione di ricordare a « initiated and the neophyte ready to be initiated of mythical precedents of initiation, of divine mystai and their experiences, and of some of the principal symbols of the Dionysiac religion 87 ". Sebbene l'originalità di Rostovtzeff non consistesse tanto nell'esegesi della megalografia quanto nell'audace tentativo di ridurre a una stretta unità tematica i dipinti della Villa e della Casa del Criptoportico, è indubbio che, per ciò che riguarda il fregio della Villa dei Misteri, le pagine di "Mystic Pompeii" attestavano lo sforzo - certamente innovativo - di contemperare e comprendere in un'unica spiegazione il tema iniziatico (e in qualche misura anagogico) e quello nuziale ${ }^{88}$, l'aspetto rituale e quello mitico, variamente enfatizzati dalla precedente letteratura. Si trattava di una posizione per così dire intermedia, consapevolmente differente da quelle decisamente unitarie e fortemente misticheggianti della

82 RostovtzefF, Mystic Italy, p. 14-16, distingueva tra il misticismo della " higher Roman aristocracy ", aperta alle rivelazioni del Neopitagorismo, quello degli " average intellectuals ", attratti dai misteri eleusini e dai misteri dionisiaci e quello, a "lower level », dei culti orientali, tutti comunque accomunati da una più o meno accentuata influenza orfica. Va ricordato che in RostovtzefF, "The Mentality", in tempi di "revisionismo" del panorfismo degli anni Venti, il tema dell'orfismo slittò in secondo piano nell'esame della religiosità ellenistica.

83 Rostovtzeff, Mystic Italy, p. 35-36 ; cf. Michelotto, “Un'opera 'anomala”, p. 55-56.

84 RostovtzefF, Mystic Italy, p. 33.

85 Zielinski, The Religion, p. 156 ; per Macchioro cf. infra.

86 Ben più orientate verso un'escatologia di tipo "orfico" erano le interpretazioni rostovtzeviane dei pinakes della Casa del Criptoportico : cf. RostovtzefF, Mystic Italy, p. 68-78 (scena di Caronte) ; 87-90 (ierogamia) ; contra Spinazzola, Pompei alla luce, p. 504-521. Cf. MichelotTo, "Un'opera 'anomala”, p. 47-52 ; 57-62.

87 RostovtzefF, Mystic Italy, p. 46.

88 Da ultimo nel volume di VEYNE, I misteri; ivi bibliografia pressoché completa nelle note a p. 273-305. 
Cooke, di Macchioro e di Rizzo ${ }^{89}$, ma comunque, in generale, più vicina ad esse che all'interpretazione realistico-mitologica di Comparetti ${ }^{90}$, sostanzialmente ripresa dal Reinach ${ }^{91}$ che nel fregio aveva riconosciuto semplicemente la rappresentazione delle nozze di Bacco e Arianna, il prototipo mitico della cerimonia nuziale. Assennatamente Rostovzeff non credeva che nel fregio della Villa e nei pinakes della Casa del Criptoportico ${ }^{92}$ fossero riprodotti direttamente e puntualmente atti e riti di iniziazione, nella convinzione - questa sì bizzarra - che solo a partire dal I sec. d. C. si sarebbe diffusa l'usanza di riprodurre esplicite (sic!) scene di iniziazione ${ }^{93}$.

Pur nella certezza che la Villa appartenesse a membri dell'aristocrazia pompeiana iniziati ai misteri dionisiaci, Rostovtzeff era ben lontano dall'identificare la " sitting room 94 " con una basilica 95 adibita alle iniziazioni, semmai preferendo attribuire questa funzione al triclinio della Casa del Criptoportico ${ }^{96}$. Destinazione della sala e interpretazione generale della megalografia creavano un diaframma sostanziale tra Rostovtzeff e Macchioro, le cui ipotesi ricostruttive tuttavia intrigavano Rostovtzeff non solo perché erano supportate da una formidabile erudizione e da un suggestivo comparativismo, ma perché il pan-orfismo di Macchioro - che, come è noto, era desti-

89 P.B. Mudie COOKE, "The paintings of the villa item at Pompeii”, Journal of Roman Studies 3 (1913), p. 157-174 ; MACCHIORO, Zagreus, 1920, p. 11 ; ID., The Villa, p. 12 ; ID., Zagreus, $1930^{2}$, p. 19 ; Rizzo, "Dionysos mystes", p. 61 (= p. 25 dell'estratto). Accomuno le posizioni - diversissime - di questi lavori perché essi convergono nel ravvisare nel complesso figurato un rituale d'iniziazione misterica, peraltro già suggerito da G. DE Petra, "Villa romana presso Pompei”, Notizie degli Scavi di Antichità Va ser., 7 (1910), p. 139-145.

90 Eccellente status quaestionis sino al 1931 in MAIURI, La Villa dei Misteri, p. 164 ; per successive "messe a punto" cf. ad es. G. GRIECO, "La grande frise de la Villa des Mystères et l'initiation dionysiaque", La Parola del Passato 188-189 (1979), p. 417-441 ; G. SAURON, La grande fresque de la Villa des Mystères à Pompéi, Paris, 1998, p. 42-52, che però, datando per una svista Mystic Italy al 1917, erroneamente colloca Rostovtzeff nella "première époque " degli studi sulla Villa.

91 S. Reinach, Répertoire des Peintures Grecques et Romaines, Paris, 1922, p. 115.

92 RostovtzefF, Mystic Italy, p. 92.

93 RostovtzefF, Mystic Italy, p. 94-98. Sulla raffigurazione dell'elmo pompeiano che Rostovtzeff poneva al centro della sua discussione cf. NILSSON, The Dionysiac Mysteries, p. 84-88; 97-98.

94 RostovtZeFF, Mystic Italy, p. 44.

95 MACCHIORO, Zagreus, $1930^{2}$, p. 152-168.

96 Rostovtzeff, Mystic Italy, p. 93 ; contra Maiuri, "Studi e ricerche”, p. 265-266 e Spinazzola, Pompei alla luce, p. 665, n. 367. Va ricordato che SPINAZzola, Pompei alla luce, p. 575-576, capovolse i risultati rostovtzeviani, nella convinzione che nessuna forma di misticismo fosse rintracciabile nella Casa del Criptoportico, a differenza che nella Villa dei Misteri, per la quale parlava addirittura di « creazione sacerdotale ». 
nato a culminare nella tormentata analisi della cristologia paolina 97 - forniva, sia pure da un'angolazione tutta particolare, una potente e coerente chiave di lettura di quella koiné mistica, di origine greca e di tradizione ellenistica, che anche Rostovtzeff vedeva diffusa tra il I a.C. e il I d.C. Solo a tratti e in maniera sostanzialmente estrinseca il simbolismo rostovtzeviano era però imparentato con il realismo mistico del "drama sacramentale " di Macchioro che, per quanto contestato e rifiutato dalla critica con maggiore o minore violenza polemica ${ }^{98}$, rimaneva - per la compattezza e la complessità dell'elaborazione teoretica - un passaggio obbligato per tutti gli esegeti, Rostovtzeff e Maiuri in testa, che in quegli anni si cimentavano nell'interpretazione della megalografia 99 .

Attingendo di volta in volta ad autori diversi (e tra loro inconciliabili come Rizzo e Macchioro), Rostovtzeff si addentrava nell'esegesi delle singole scene : ad esempio tentava di rinvenire una connessione tra la figura alata di un pinax della Casa "Omerica" e il celeberrimo "demone alato" della megalografia. Qui l'analogia con l'esegesi di Macchioro si limitava all'identificazione del "demone alato" con Teleté, in quanto Rostovtzeff rifiutava la communis opinio (seguita anche da Macchioro) della flagellazione più o meno rituale, allineandosi alla posizione, allora decisamente minori-

97 Macchioro, Zagreus, $1930^{2}$, p. 289-540. Qui confluirono saggi del 1923 e del 1928 (si veda la prefazione in Macchioro, Zagreus, $1930^{2}$, p. 5). Cf. R. Di DonAto, "Preistoria di Ernesto De Martino", Studi Storici 30 (1989), p. 225-246, in particolare p. 231.

98 Lo Zagreus suscitò reazioni particolarmente dure negli ambienti degli archeologi italiani : cf. MAIURI, La Villa dei Misteri, p. 218, n. 49. Ben diversa l'accoglienza presso alcuni filosofi e storici delle religioni : Di DONATO, "Preistoria", p. 230 riporta un lungo stralcio della " attenta e favorevole recensione » di Giovanni Gentile al primo Zagreus (del 1920) e ricorda (p. 233) che "il giovane Mircea Eliade includeva l'autore di Zagreus tra i suoi idoli intellettuali ed avviava una corrispondenza che continuerà fino al 1939 ».

99 Cf. J. ToYnBeE, "The Villa Item and a Bride’s Ordeal", Journal of Roman Studies 19 (1929), p. 67-87. Riprese della discussione sulle singole scene in F. MATZ, " $\Delta I O N Y \Sigma I A K H$ TE $\Lambda$ ETH. Archäologische Untersuchungen zum Dionysoskult in hellenistischer und römischer Zeit", Abhandlungen der Akademie der Wissenschaften in Mainz 15 (1963), p. 13871454 (= p. 3-70 dell'estratto) ; G. ZunTZ, "On the dionysiac fresco in the Villa dei Misteri at Pompeii", Proceedings of the British Academy 49 (1963), p. 177-201 ; H. JEanmaire, Dioniso. Religione e cultura in Grecia, traduzione italiana, Torino, 1972, p. 387 ; 457 ; M.L. West, I poemi orfici, traduzione italiana, Napoli, 19932, p. 37 ; K. KerÉNYI, Dioniso. Archetipo della vita indistruttibile, traduzione italiana, Milano, 1993, p. 325 ; M. GuARDuCCI, "Dioniso e la cosiddetta Villa dei Misteri a Pompei", Rendiconti dell'Accademia dei Lincei 8.9, V.4 (1993), p. 521-533 ; B. HundSAlZ, "Neues zum grossen Fries der Mysterienvilla”, Kölner Jahrbuch 24 (1991), p. 73-78. Per i dettagli rimando a MichelotTo, "Un'opera 'anomala”, p. 58-62. 
taria ${ }^{100}$, del suo amico S. Reinach che - a proposito della pletora di interpretazioni offerte sulla " prétendue flagellation rituelle » - non aveva esitato ad affermare : «On a écrit des insanités 101. " Rostovtzeff ravvisava nella dea alata della Casa del Criptoportico ora (correttamente) « a Victory, a Nike 102 ", recante " a little hammeror key-like instrument in her right 103 ", ora - non senza ambiguità - " the goddess of initiation, Telete, or the goddess of Victory 104 " (corsivo mio), riuscendo ancora una volta, grazie a un forzato slittamento interpretativo, a saldare le rappresentazioni della Casa del Criptoportico e quelle della Villa dei Misteri.

Nell'elitario misticismo documentato a Pompei dalla Villa dei Misteri o dalla Casa "Omerica" e a Roma dalla Casa della Farnesina o dalla "Basilica neopitagorica" presso P.ta Maggiore si rifletteva il più ampio disagio proprio di "an age of anxiety", secondo il topos per cui il misticismo era un portato delle epoche di crisi economico-sociale, delle epoche di guerra e di rivoluzione. Si trattava in ogni modo di un fenomeno che comportava irreversibili mutazioni di mentalità e si traduceva in processi di « degene-

100 Rostovtzeff, Mystic Italy, p. 54 attribuiva " apotropaic gesture " al "demone" ; cf. MACCHIORO, Zagreus, 1930², p. 129-130 e già CoOKe, “The Paintings”, p. 166. Rassegne relative alle diverse interpretazioni in NiLSSON, The Dionysiac Mysteries, p. 123-125; MATZ, “ $\triangle \mathrm{ION}$ YIIAKH TE $\Lambda$ ETH”, p. 1406-1408 (= p. 22-24 dell'estratto), ZUNTZ, “On the Dionysiac", p. 194-197 ; R. TURCAN, "La démone ailée de la Villa Item”, in J. Bibauw (a cura di), Hommages à Marcel Renard, I-III (Collection Latomus, 103), Bruxelles, III, 1969, p. 586-609 ; ID., Les cultes orientaux dans le monde romain, Paris, 1992², p. 305310 ; GRIECO, "La grande frise", p. 427-431 ; A. BARBET, La peinture murale romaine : les styles décoratifs pompéiens, Paris, 1985, p. 52-53 ; W. BURKERT, Antichi culti misterici, traduzione italiana, Roma-Bari, 1991, p. 137-139 ; 189, n. 90 ; S. ADAmO MuSCETTOLA, "La trasformazione della città tra Silla e Augusto", in F. ZEVI (a cura di), Pompei, I-II, Napoli, I, 1991, p. 75-114, in particolare p. 103 ; VEYNE, I misteri, p. 83-91 ; 294, nn. 220-224 ; SAURON, La grande fresque, p. 94-106. Per il rifiuto, in tempi più recenti, della teoria della flagellazione cf., oltre ai sopra menzionati lavori di Turcan e Guarducci, K. LEHMAnN, "Ignorance and search in the Villa of the Mysteries", Journal of Roman Studies 52 (1962), p. 62-68, in particolare p. 65.

101 REINACH, Répertoire, 1922, p. 115.

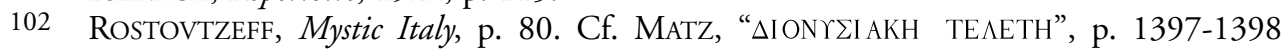
(= p. 13-14 dell'estratto). Si noti la perentorietà nella definizione delle singole scene : " divinazione ", " iniziazione ", " rivelazione " etc.

103 Rostovtzeff, Mystic Italy, p. 78 ; 81, optava alla fine (erroneamente) per la chiave. Anche SpINAZzola, Pompei alla luce, p. 517 ; 667, n. 383 ; 668-669, n. 393, pur polemizzando con Rostovtzeff che, a suo modo di vedere, "trascendeva " il significato mitico della scena, interpretava il personaggio alato come una Victoria, recante il martello e lo stilo. In questo senso, la successiva identificazione, da parte di Nilsson, Dionysiac Mysteries, p. 116 ; 124 130, del personaggio alato con Dike kleidouchos rappresentò un arretramento rispetto alle intuizioni di Rostovtzeff.

104 RostovtzefF, Mystic Italy, p. 84. 
razione psicologica 105 » destinati a produrre radicali metabolaí e, al limite, veri e propri trapassi di civiltà. Risultava difficile a Rostovtzeff stabilire con esattezza modi, tempi, luoghi in cui si realizzarono le trasformazioni e le osmosi spirituali determinate dalla diffusione di un sentimento religioso fondato sulla fede in un destino personale ultramondano : egli era imbarazzato nel definire la portata dell'influenza dei culti orientali 106 (sui quali era stato perentorio nel III capitolo de La nascita dell'impero romano), così come tentennava sulla cronologia della formazione di quella che chiamava "koiné mistica ", talora facendola risalire alla piena età ellenistica, talaltra ritenendola una conseguenza del clima infuocato e deprimente delle guerre civili a Roma e quindi rimandandola all'età augustea o, come accadeva in "Mystic Rome", a " the late First, the Second and the early Third Centuries after Christ, the quiet and somnolent time of the ripe Roman Empire 107 "; in ogni caso, ovviamente, la trasfigurazione della cultura e della spiritualità antiche doveva trovare compimento ("religion and mysticism triumphed over all ») in conseguenza del «bloody crash » del III secolo d.C. ${ }^{108}$. La sordina posta dal Rostovtzeff di "Mystic Italy" (saggio I) sull'età ellenistica era funzionale all'affermazione del modello - presente anche in The Social and Economic History of the Roman Empire - delle due crisi del mondo romano in corrispondenza delle lotte civili e rivoluzionarie del I secolo a.C. e del III secolo d.C. : modello che, come è noto, fu fieramente respinto nel 1933 da Kovalev ${ }^{109}$.

Oltre che dall'incertezza indotta da una documentazione elusiva ${ }^{110}$, le oscillazioni di Rostovtzeff dipendevano dall'assunzione di modelli di volta in volta diversi (e tra loro difficilmente conciliabili) a spiegazione delle epoche di "trapasso" e determinavano variazioni anche sensibili nell'interpretazione di momenti-cardine della storia mediter-

105 RostovtzefF, "Il tramonto della civiltà antica", p. 91-92; 126 ; 135-155; ID., Storia economica e sociale dell'Impero romano (Nuova edizione, accresciuta di testi inediti, a cura di A. MarCone), Milano, 2003, p. 733-739 ; 776-784 ; cf. P.G. MichelotTo, "La riflessione storico-economica di M.I. Rostovtzeff : il 'caso' dell'Egitto ellenistico-romano", Memorie dell'Istituto Lombardo 41, 1 (1999), p. 1-152, in particolare p. 77-83 ; ID., "Italia 1923 ”, p. 434 e lo scritto rostovtzeviano ivi pubblicato a p. 450-453. Nella " degenerazione psicologica " l'aspetto religioso rimaneva l'unico per il quale Rostovtzeff non osava pronunciare un giudizio (negativo) di valore.

106 Rostovtzeff, Mystic Italy, p. 32 ; 110-111 ; ancora in RostovtzefF, “The Mentality”, p. 23-24.

107 RostovtzefF, Mystic Italy, p. 144. RostovtzeFf, “The Mentality”, p. 15, avrebbe instaurato un parallelo tra la spiritualità della tarda epoca ellenistica e quella del primo impero romano.

108 RostovtzefF, Mystic Italy, p. 21 ; ID., "Il tramonto della civiltà antica”, p. 153-154.

109 Ho letto il contributo di Kovalev in M. RASKOLNIKOFF, La recherche en Union Soviétique et l'histoire économique et sociale du monde hellénistique et romain, Strasbourg, 1975, p. 297-303.

110 Rostovtzeff, "The Mentality”, p. 23. 
ranea quali il periodo ellenistico o l'èra di Augusto o la metamorfosi tardoantica ${ }^{111}$. Affliggevano la teoresi rostovtzeviana alcune insanabili aporie, la prima delle quali era proprio rappresentata dalla valutazione complessiva dei fattori religiosi, mai definiti come disvalori pur essendo presentati come motivo di disgregazione della civiltà classica e come elementi costitutivi della "degenerazione psicologica" dell'antichità. $\mathrm{Ne}$ conseguiva che Rostovtzeff, pur essendo convinto che tutti gli aspetti di una civiltà, da quelli economico-sociali a quelli culturali, si componessero in quadri unitari di ascesa e di declino, e cioè che ogni civiltà presentasse contemporaneamente in tutte le sue manifestazioni momenti di vitalità e momenti di eclissi, avvertiva la peculiare eccentricità - e la "sfasatura cronologica" - dei fenomeni religiosi rispetto agli altri fenomeni culturali, giungendo ad affermare che « i periodi di maggiore rigoglio nel campo della creatività religiosa quasi mai si identificano con i periodi di attività produttiva nei restanti campi della cultura; si può dire piuttosto che la creatività religiosa o precede, o segue un più alto sviluppo della creatività negli altri campi 112 ".

Una seconda aporia, intrecciata alla prima, consisteva nel far convivere una concezione fortemente negativa, "belochianamente" distruttiva, dell'intervento romano in Oriente e la convinzione - irrinunciabile per Rostovtzeff - che con l'esperienza imperiale romana si fosse aperto non solo, genericamente, un "real progress in the history of civilization ", ma una nuova fase, un nuovo e originale aspetto della cultura e della spiritualità ellenistiche ${ }^{113}$. La compresenza di una pluralità di modelli interpretativi conduceva Rostovtzeff a singolari contraddizioni nella valutazione qualitativa della cultura romana in generale e in particolare del mondo augusteo ${ }^{114}$.

Rispetto a "Mystic Pompeii" e a "Mystic Rome", imperniate sull'esame di documenti archeologici, "Mystic Italy" (saggio I) palesava la sua origine di conferenza di natura "teorica" e polemica, in cui l'antico e il moderno (la moderna storia russa) si intrecciavano assiduamente sulla base di un veemente registro espressivo quale neppure si rinveniva né ne La nascita dell'impero romano ${ }^{115}$ né nelle feroci tirate antimateriali-

111 Michelotto, "La riflessione storico-economica”, p. 77-86; 105-118.

112 RostovtZeFF, "Il tramonto della civiltà antica", p. 91. Naturalmente questa concezione si prestava a una rivalutazione del periodo tardoantico ; recentemente ne ho parlato in Rivista Storica Italiana 116 (2004), p. 211-229, recensendo l'edizione della Storia economica e sociale dell'Impero romano curata da Arnaldo Marcone.

113 Rostovtzeff. Mystic Italy, p. 9 ; 15-16. Cf. Rostovtzeff, "Il tramonto della civiltà antica", p. 135-155 ; RostovtzefF, "The Mentality", p. 5-6. Anche nel cap. III de La nascita dell'impero romano Rostovtzeff si dilungava su questo tema.

114 MichelotTO, "La riflessione storico-economica", p. 73-83.

115 Cf. supra n. 3. Wes, Michael Rostovtzeff, p. 66-74 ; ANDrEaU, "Antique, moderne", p. XXI-XXII ; RASKOLNIKOFF, La recherche, p. 33-34. 
stiche del 1930 116. A "Mystic Italy" (saggio I) come a pochi scritti rostovtzeviani si attaglia quanto icasticamente espresso da Jean Andreau : "L'histoire ancienne est pour lui un substitut librement choisi de l'histoire de la Russie, qui eût elle-même été un substitut de l'action politique ${ }^{117}$. " Nello zielinskiano miroir dell'antico ${ }^{118}$, Rostovtzeff vedeva delinearsi il drammatico destino di fallimento di qualsiasi forma (elitaria o popolare) di materialismo e delle dottrine su di esso costruite, a cominciare da quelle socialiste e comunistiche già ben note - sosteneva Rostovtzeff - al mondo classico al tempo della ottimistica espansione delle sue energie spirituali. Nello smisurato orgoglio - e nell'improvviso crollo - del razionalismo scientista greco ed ellenistico (che qui veniva considerato, a differenza di quanto sarebbe accaduto in "Mystic Pompeii" e in "The Mentality", un blocco unitario sostanzialmente a-religioso), nell'inguaribile arroganza di una élite da cui " religion and a religious conception of life were regarded as old-fashioned and reactionary 119 ", e in particolare nell'ottuso scetticismo di quegli intellettuali atei o al più vagamente deisti come il primo Cicerone - sempre comunque recidivi nella derisione della fede popolare e inclini a reputare il sentimento religioso delle masse come " instrument of domination 120 " - si riflettevano lo stesso altezzoso distacco dalle masse, lo stesso cieco illuminismo, lo stesso drammatico destino del-

116 M. RostovtzefF, "The decay of the ancient world and its economic explanations", The Economic History Review II 2 (1930), p. 197-214 = traduzione italiana in ROSTOVTZEFF, Per la storia economica e sociale, p. 199-218 ; cf. P.G. MichelotTo, "La History di Rostovtzeff dal 1926 al 1933 : intorno ad alcune varianti”, in MARCONE (a cura di), Rostovtzeffe l'Italia, p. 187-209 ; MiCHELOTTO, “Un'opera 'anomala”, p. 64-73.

117 J. ANDREAU, "M.I. Rostovtseff, la décadence romaine et la révolution russe", in J.-M. PAiller (a cura di), Actualité de l'Antiquité, "Actes du Colloque organisé à l'université de Toulouse-Le Mirail par la revue PALLAS - Décembre 1985”, Paris, 1989, p. 27-33, in particolare p. 32 ; ID., "La dernière des grandes synthèses historiques de Michel Ivanovič Rostovtseff", "Introduction" a M.I. RosTOVTSEFF, Histoire économique et sociale $d u$ monde hellénistique, traduction française, Paris, 1989, p. I-XXIX ; cf. B.D. SHAW, "Under Russian Eyes", Journal of Roman Studies 82 (1992), p. 216-228.

118 T. ZIELINSKI, L'Antico e Noi, traduzione italiana, Firenze, 1910 ; esempio significativo in Zielinski, La Sibylle, p. 57-58. Cf. MichelotTo, "La riflessione storico-economica", p. 18.

119 Rostovtzeff, Mystic Italy, p. 3-4. Per l'ellenismo inteso come epoca profondamente religiosa cf. ad es. Rostovtzeff, Mystic Italy, p. 35-37 ("Mystic Pompeii" ; ID., Storia del mondo antico, traduzione italiana, Firenze, 1975, p. 369-385 ; ID., "Il tramonto della civiltà antica", p. 142-155. Il RostOvTZEFF di "The Mentality", riprendendo con maggiore pacatezza il tema della parabola del razionalismo greco, evitava il problema dell'eventuale declino della scienza che è invece assolutamente centrale in "Mystic Italy" (saggio I).

120 Rostovtzeff, Mystic Italy, p. 5. Da rilevare che in "Mystic Italy" (saggio I) Rostovtzeff attribuiva questo scettico e cinico atteggiamento anche ai sovrani ellenistici. Diversissimo il giudizio in "Mystic Pompeii" (RostovtzefF, Mystic Italy, p. 35-36) e già in RostovTZEFF, "Il tramonto della civiltà antica", p. 143-145. 
l'intelligencija russa dopo il rovinoso fallimento, culturale prima che politico, delle rivoluzioni del 1905 e del febbraio 1917. Proprio nella figura di Cicerone ${ }^{121}$, "the best man of this time 122 ", " una delle migliori espressioni dell'epoca rivoluzionaria 123 ", non solo si condensavano le contraddizioni e l'impotenza politica che affliggevano anche gli intellettuali russi ${ }^{124}$, ma si prefigurava un itinerario di conversione ${ }^{125}$ dallo scetticismo al misticismo simile a quello percorso da una cospicua frazione dell' intelligencija russa e attestato, tra l'altro, dalle raccolte di Vekhi (1909) e, soprattutto, di $I z$ glubiny (= Dal profondo, 1918) ${ }^{126}$.

Tipico del Rostovtzeff polemista, lo schematismo di "Mystic Italy" (saggio I) lasciava zone d'ombra su temi fondamentali (come quello - destinato a divenire l'argomento-cardine di "The Mentality" - delle lente mutazioni psicologiche e religiose veri-

121 Naturalmente il Cicerone di Rostovtzeff era molto lontano dall' "avvocato liberale” raffigurato nella primavera del 1918 da A. BLOK, "Catilina”, in BLOK, Lintelligencija e la rivoluzione, traduzione italiana, Milano, 1978, p. 75-117, in particolare p. 92. RosTOVTZEFF, Mystic Italy, p. 16 ; cf. p. 5.

123 Rostovtzeff, La nascita, in c. di s. Cf. Rostovtzeff, Storia del mondo antico, p. 521 ; 549.

124 RostovtzefF, La nascita, in c. di s. Cito dalla traduzione di A. Ferrari attualmente in corso di stampa (cf. supra n. 3) : "In politica egli servì sempre un unico scopo fondamentale, il bene di Roma. L'incoerenza, talvolta anche la codardia, l'aspirazione a perseguire interessi personali... non può celarci il vero volto di questo autentico patriota, il cui difetto principale era di essere un intellettuale puro, un tipico rappresentante dell' humanitas dell'antichità ".

125 RostovtzeFF, Mystic Italy, p. 17.

126 Dal profondo (Iz glubiny). Raccolta di saggi sulla rivoluzione russa, traduzione italiana, Milano, 1971 ; La svolta. Vechi. L“'intelligencija” russa tra il 1905 e il '17, traduzione italiana, Milano, 1990. Cf. Wes, Michael Rostovtzeff, p. 63-74 ; MichelotTo, "Un'opera 'anomala”, p. 64-73 (ivi ulteriori riferimenti bibliografici). Non c'è spazio per riprendere qui il problema dei rapporti tra Rostovtzeff e gli intellettuali di Vekhi e di Iz glubiny : le indubbie consonanze (in particolare con Frank e con Struve) e la compartecipazione a un "clima" psicologico e culturale non devono far dimenticare le profonde, sostanziali differenze : mai - neppure al tempo del soggiorno inglese e nei primi anni di Madison - il pensiero storico di Rostovtzeff fu assimilabile o riducibile alle teorizzazioni filosofiche (Rostovtzeff non amava la filosofia della storia : cf. J. ANDREAU, recensione a WeS, Michael Rostovtzeff, in TOПOI 4, 1 (1994), p. 281-283 ; MICHELOTTO, La riflessione storico-economica, p. 10-11) e psico-sociologiche degli intellettuali di Iz glubiny. Nel 1923, mentre Rostovtzeff formulava sinteticamente nel Musée Belge la sua notissima teoria della decadenza (M. RostovtzeFf, "La crise sociale et politique de l'Empire romain au IIIe siècle", Musée Belge 27 (1923), p. 233-242 = traduzione italiana in RostovtzefF, Per la storia economica e sociale, p. 157-165), Berdjaev pubblicava Il senso della storia : persino a una lettura superficiale dei due testi, risulta evidente quanto sia differente la natura del pessimismo in entrambi presente ; per inciso, è allo stesso modo fuorviante qualsiasi diretto riferimento a Spengler, pace Sergeev : cf. RASKOLNIKOFF, La recherche, p. 59. Su Berdjaev cf. A. Giustino Vitolo - G. Lami, Storia e filosofia in N.A. Berdjaev, Milano, 2000. 
ficatesi in età ellenistica) e, d'altro canto, impediva una lettura nel contempo sfumata e coerente delle conseguenze dell'intervento romano in Oriente, vale a dire del problema del continuum ellenistico-romano e in particolare del momento augusteo, che dai punti di vista politico, spirituale e culturale costituì il floruit dell'eredità ellenistica in Roma. Dopo aver sottolineato il carattere di "originalità nella continuità" della cultura latina, specie nelle sue espressioni artistiche e letterarie 127, Rostovtzeff sorprendentemente negava all'età augustea la capacità di produrre " new genres, new literary forms, new and inspiring ideas 128 " e ravvisava la stessa mancanza di creatività, oltre che nella scienza, anche nell'arte del I e II d.C. ${ }^{129}$. In modo simile, nelle pagine finali della Storia del mondo antico, Rostovtzeff sosteneva che "l'affermarsi dell'impero fu accompagnato da nuovi progressi del genio creativo ", ma si affrettava ad aggiungere : "A questo progresso mancava l'entusiasmo e la forza che avevano contraddistinto l'opera delle città greche ed anche della Roma repubblicana. Fin dagli inizi vi si notano i segni della stanchezza e della disillusione, segni caratteristici di un'epoca post-rivoluzionaria ; e più tardi, nella calma atmosfera della pace... scompare ogni energia vitale ${ }^{130}$. "

In "Mystic Italy" (saggio I) Rostovtzeff riconosceva al programma politico interclassista di Augusto (semplicisticamente riassunto nella formula " to reconcile the aspirations of rich and poor ") il merito di aver restaurato l'ordine e la pace e di aver aperto " a golden age 131 » destinato a protrarsi sino al III secolo, ma della pax augustea sottolineava l' " artificial atmosphere " che veniva a sovrapporsi a un diffuso stato d'animo di stanchezza, di pessimismo e di noia : "And yet the general mood of the people, upper and lower classes alike, was world-weary ${ }^{132}$."

Come la politica augustea, tanto sensibile nel captare il sentimento dominante nelle masse ${ }^{133}$, così la grande poesia dell'epoca derivava il suo successo dalla capacità

127 RostovtzefF, Mystic Italy, p. 9.

128 Ripresa di concetti più o meno contraddittori già formulati in RosTOVTZEFF, La nascita, in c.di s. ; ID., Storia del mondo antico, p. 715 ; ID., "Il tramonto della civiltà antica", p. $138-141$.

129 RostovtzefF, Mystic Italy, p. 18. La contraddizione fu notata già da FLICKINGER, p. 154, nella sua recensione al libro, per cui cf. supra n. 60. In RosTOVTZEFF, "Il tramonto della civiltà antica", p. 117, si parlava dell' " enorme creatività " dispiegata dall'impero romano «nel campo della letteratura e dell'arte nel primo secolo e mezzo della sua esistenza ».

130 RostovTZEFF, Storia del mondo antico, p. 715.

131 RostovtzeFf, Mystic Italy, p. 17 ; 21 (dove il «blessed period » viene attribuito a merito anche dei successori di Augusto). Cf. M. RostovtZefF, s.v. "Augusto", Nuovo Dizionario Enciclopedico Brockhaus-Efron, I, S. Pietroburgo, 1911, col. 152-165 (in russo), in particolare col. 162.

132 RostovtzefF, Mystic Italy, p. 18-19. Cf. ID., La nascita, in c. di s. ; ID., Storia del mondo antico, p. 587-597.

133 RostovTZEFF, La nascita, in c. di s. ; ID., Storia del mondo antico, p. 567 ; 591-593 ; ID., "Augustus" 1922, p. 134-148 e il capitolo II di The Social \& Economic History of the Roman Empire, Oxford, 1926, p. 38-74 = Nuova edizione italiana curata da MARCONE, p. 47-102. 
di rendersi interprete dei bisogni spirituali e delle aspirazioni delle masse, delle vaghe quanto diffuse idee messianiche nutrite dall'angoscia e dalla stanchezza delle guerre civili ; ne La nascita dell'impero romano Rostovtzeff era giunto ad affermare : " Non i poeti suggerivano alle masse pensieri ad esse estranei e nuovi, ma piuttosto il contrario : erano le masse ad influire sui poeti con i loro sentimenti ed umori, forse informi ed inconsapevoli ${ }^{134}$. " All'interno del più generale dilemma se e in che misura un'autentica religiosità personale, che Rostovtzeff considerò sempre come un'estraniazione dell'individuo dalla sfera dello Stato, si prestasse ad essere recepita e, per così dire, assimilata da forme ed espressioni "ufficiali" di culto, si apriva in particolare la questione se " the mystical mood of the population 135 " avesse trovato spazio e consonanza nel programma augusteo di rinnovamento morale e di restaurazione/riforma religiosa che nel culto imperiale aveva il suo fulcro. Il problema presentava numerose articolazioni che per Rostovtzeff costituivano altrettanti, spinosi, punti interrogativi ai quali non seppe mai offrire risposte univoche e decisive.

Ne La nascita dell'impero romano, guardando in controluce le lotte triumvirali e vedendo, come sua consuetudine, la filigrana della rivoluzione russa, Rostovtzeff aveva ravvisato in Augusto la lucida capacità di condurre a un raffreddamento per così dire "termidoriano 136" la magmatica ondata rivoluzionaria dell'esercito mediante un programma politico almeno apparentemente interclassista e mediante un'abile politica culturale e religiosa volta a convogliare, e a "sfruttare 137 ", il generale desiderio di pace e le speranze messianiche in vario modo nutrite dalle masse italiche. A questa prospettiva Rostovtzeff apportava qualche significativo ritocco nei quadri complessi e non sempre armonici de "Il tramonto della civiltà antica" e della Storia del mondo antico : malgrado il "rivoluzionario e politico Augusto " fosse " troppo poco simile ad un Salvatore ", personalmente privo financo di « una goccia di sincero romanticismo 138 " e la sua "figura... così prosaica e così lontana dal divino 139 " da ricevere solo l'occasionale e freddo omaggio della poesia contemporanea ${ }^{140}$, la schietta venerazione e la corale gratitudine delle masse favorirono - forse al di là delle intenzioni dello stesso

\footnotetext{
134 RostovtzeFF, La nascita, in c. di s.

135 RostovtzeFF, "Augustus" 1922, p. 139.

136 In generale cf. D.V. SHLAPENTOKH, "Thermidor or Mongol Empire : History as Political Model in Russian Émigré Thought", Cahiers du Monde Russe et Soviétique 32, 3 (1991), p. 379-408.

137 RostovTZEFF, La nascita, in c. di s.

138 RostovtZEFF, "Il tramonto della civiltà antica", p. 146 e p. 93.

139 RostOvTZEFF, Storia del mondo antico, p. 591.

140 RostovtzeFF, "Il tramonto della civiltà antica", p. 146 : « Ma in tutto questo movimento non c'è uno slancio sincero, una fede vera nella forza creativa della nuova era... ". Più articolato il giudizio sulla poesia vergiliana in ROSTOVTZEFF, Storia del mondo antico, p. 596597 ; cf. ID., “Augustus" 1922, p. 141-142.
} 
princeps ${ }^{141}$ - la costituzione di un culto che inizialmente 142 ebbe il carattere di una "vera religione " appassionatamente vissuta, prima di recedere, come Rostovtzeff scriveva in "Mystic Rome", al rango di " a convention, a formula, a tradition, an abstraction " e cioè di una mera sovrastruttura 143 comparabile agli altri culti ufficiali "di Stato", in particolare a quello della Triade Capitolina. Proprio in "Mystic Rome", tuttavia, riprendendo a tinte sfumate alcuni temi dell' "Augustus" del 1922, Rostovtzeff si soffermava sulla complessità e sulle potenzialità del culto imperiale : sostanziato dal clima di diffuso misticismo e di generale attesa messianica, il culto imperiale - «strange

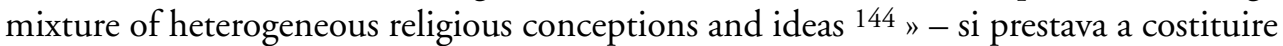
una sorta di bacino collettore del consenso e un momento unificante di credenze di varia origine (italica, ellenistica), di varia natura e di vario livello intellettuale. In forme ancor più accentuate che in "Mystic Rome", nel precedente "Augustus", in cui appariva l'ampio spettro di significati politici e religiosi delle riforme augustee, Rostovtzeff non solo aveva sottolineato, come d'abitudine, la capacità di Augusto di porsi come geniale interprete della diffusa ansia di rinnovamento spirituale ("Augustus as a religious reformer !»), ma anche aveva enfatizzato la personale fede del princeps nell'astrologia 145 , oltre che una romantica e nostalgica esigenza di recupero delle credenze dei padri ; esigenza caratteristica delle epoche postrivoluzionarie in cui tendono a combinarsi e a confluire istanze razionalistiche e " old-fashioned religiosity " - aveva aggiunto Rostovtzeff, con un occhio agli orientamenti della intelligencija romana del I secolo a.C. (in particolare del "circolo" di Mecenate) e con l'altro alle tendenze della intelligencija russa del suo tempo ${ }^{146}$.

141 RostovtzefF, "Il tramonto della civiltà antica", p. 93 : Augusto non ebbe " neanche un'ombra di convinzione nella propria divinità : Augusto non rivendicò per sé onori divini ». Cf. però RostovtzefF, Storia del mondo antico, p. 591-597.

142 RostovtzeFF, "Il tramonto della civiltà antica", p. 92-93, affermava che non solo Augusto, ma anche i suoi immediati successori "divennero oggetto di un caloroso sentimento religioso e di sincera venerazione "; così pure in "Mystic Rome" (RosTOVTZEFF, Mystic Italy, p. 106-107) in cui però precisava : "In the long run, however, it was impossibile to maintain warm religious feelings towards a succession of men who had so little of divine in them " (p. 108) ; cf. ID., "Augustus" 1922, p. 145 ; ID., Storia del mondo antico, p. 587-597.

143 RostovtZeFf, Mystic Italy, p. 108 ; ID., "Il tramonto della civiltà antica”, p. 152.

144 RostovtzeFf, Mystic Italy, p. 104 ; cf. p. 107-108.

145 RostovtzefF, "Augustus" 1922, p. $134 ; 137$. Rostovtzeff traeva la notizia della conversione di Augusto all'astrologia (mai da lui altrove enfatizzata) da Astrology and Religion di Cumont (1912 = F. Cumont, Astrologia e religione presso i Greci e $i$ Romani, traduzione italiana, Milano, $1997^{2}$, p. 123), come risulta inequivocabile dall'immediatamente successivo riferimento al misticismo posidoniano evocato da Cumont e ripreso puntualmente da Rostovtzeff che, a proposito di Posidonio, sempre si attenne - come Cumont - alla vulgata interpretativa di ascendenza schmekeliana (l'opera di Schmekel è del 1892).

146 RostovtZEFF, "Augustus” 1922, p. 135-136; 138. Valutazione opposta in RosTOVTZEFF, "Il tramonto della civiltà antica", p. $93 ; 146$. 
Nelle continue oscillazioni di Rostovtzeff nella valutazione del culto imperiale si rifletteva il più generale e profondo disagio determinato dall'incertezza nell'inquadrare la politica di Augusto. Come sarebbe accaduto nel tormentato esame della rinascenza tardoantica ${ }^{147}$, anche a proposito del valore storico del floruit augusteo Rostovtzeff si trincerava imbarazzato dietro il concetto di "breve durata ", ben presente nel l'AAugustus 148" come nei saggi di Mystic Italy 149.

In seguito Rostovtzeff preferì evitare di misurarsi di nuovo con il tema della religiosità dell'epoca augustea : già relegato in secondo piano in "Mystic Pompeii", esso fu trattato con un certo distacco in The Social and Economic History of the Roman Empire ${ }^{150}$. Più in generale, specifici ritorni ad argomenti religiosi furono infrequenti e occasionali, come avvenne per la conferenza del 1938, “The Mentality”. È a questo proposito significativo che in "The Decay of the Ancient World" del 1930 151, in cui riprendeva la polemica antimaterialistica, antilinearista e antieconomicistica in rapporto al tema della decadenza, Rostovtzeff non accennasse minimamente al problema religioso. Ma - e qui si palesa l'indissolubile intreccio dei "fuochi di ispirazione" che stavano alla base di Mystic Italy - l'attenuazione dell'interesse per gli argomenti religiosi (che fu vivo tra il 1918 e il 1925 e vivissimo tra il 1922 e il 1924) si accompagnò in Rostovtzeff a una diminuita attenzione per le guerre civili del I secolo a.C. e per il connesso problema dell'interpretazione della temperie spirituale dell'età augustea. Parallelamente - a conferma di quanto profondamente la storia presente incidesse nella trattazione dei temi sopra indicati -, dopo gli anni di Madison (ma direi già dopo la recensione del 1923 a Russia To-day and To-morrow di Miliukov 152), crollava

147 Ne ho parlato in MichelotTo, "La riflessione storico-economica", p. 111-122 e in Rivista Storica Italiana 116 (2004), p. 225-228.

148 RostovtZeFF, "Augustus" 1922, p. 145-146.

149 RostovtzeFf, Mystic Italy, p. 38 ; 108.

150 Rostovtzeff, The Social \& Economic History, p. 43 : " half-religious awe " = Nuova edizione italiana curata da MARCONE, p. 53.

151 RostovtzefF, The Decay of the Ancient World, 1930, p. 197-214 = traduzione italiana in RostovtzefF, Per la storia economica e sociale, p. 199-218. Cf. Michelotto, "La History di Rostovtzeff", p. 203-209.

152 M. RostovtzefF, "A Russian Statesman”, recensione a P.N. Miliukov, Russia To-day and To-morrow, New York, 1922, in The Yale Review 12 (1923), p. 425-428. 
la produzione rostovtzeviana di saggi e articoli sulla Russia contemporanea 153 . Rimaneva acceso il "fuoco di ispirazione" più antico e tenace : l'archeologia. L'avventura di Dura-Europos attendeva il "pompeiano" Rostovtzeff.

Pier Giuseppe MichelotTo

Università degli Studi di Milano

Via Verona, 3

I-20048 Carate Brianza (Italia)

aizani@tinoit; piergiuseppe.michelotto@unimi.it

153 Dei 34 lavori rostovtzeviani sulla Russia moderna elencati da WeLLES, "Bibliography" p. 380-381, solo tre sono posteriori al 1923 e solo uno posteriore al 1926. 